\title{
Complexity growth with Lifshitz scaling and hyperscaling violation
}

\section{Mohsen Alishahiha, ${ }^{a}$ Amin Faraji Astaneh, ${ }^{b, c}$ M. Reza Mohammadi Mozaffar ${ }^{a}$ and Ali Mollabashi ${ }^{a}$}

${ }^{a}$ School of Physics, Institute for Research in Fundamental Sciences (IPM), P.O. Box 19395-5531, Tehran, Iran

${ }^{b}$ Physics Department, Faculty of Sciences, Arak University, Arak 38156-8-8349, Iran

${ }^{c}$ School of Particles and Accelerators, Institute for Research in Fundamental Sciences (IPM), P.O. Box 19395-5531, Tehran, Iran

E-mail: alishah@ipm.ir, faraji@ipm.ir,m-mohammadi@ipm.ir, mollabashi@ipm.ir

ABSTRACT: Using "complexity=action" proposal we study the growth rate of holographic complexity for Lifshitz and hyperscaling violating geometries. We will consider both one and two sided black branes in an Einstein-Maxwell-Dilaton gravitational theory. We find that in either case Lloyd's bound is violated and the rate of growth of complexity saturates to a value which is greater than twice the mass of the corresponding black brane. This value reduces to the mass of the black brane in the isotropic case. We show that in two sided black brane the saturation happens from above while for one sided black brane it happens from below.

KeYwords: AdS-CFT Correspondence, Black Holes, Gauge-gravity correspondence, Space-Time Symmetries

ARXIV EPRINT: 1802.06740 


\section{Contents}

1 Introduction 1

2 Holographic complexity for black branes $\quad 2$

3 Holographic complexity for Vaidya metric $\quad 10$

4 Conclusions $\quad 14$

\section{Introduction}

In the context of gauge/gravity duality the holographic entanglement entropy [1] has provided a geometric (classical) description of a quantum mechanical object. This correspondence might indicate that there could be a deep relation between quantum gravity and quantum information theory. If this is correct, one may expect for instance that the nature of space time geometry could be understood from quantum information theory. It would be, then, interesting to understand quantum information theory holographically, in the sense that there could be a holographic dual for some quantum information theory objects.

Actually, the computational complexity could be thought of as an explicit example for this general idea. Indeed based on earlier works of $[2,3]$, it was proposed that for a theory with a gravitational dual, the complexity of a holographic boundary state can be identified with the on-shell action evaluated on a certain subregion of the bulk space time. More precisely, in this proposal which is known as the 'complexity=action' (CA), the quantum computational complexity of a holographic state is given by the on-shell action evaluated on a bulk region known as the 'Wheeler-De Witt' (WDW) patch $[4,5]^{1}$

$$
\mathcal{C}(\Sigma)=\frac{I_{\mathrm{WDW}}}{\pi \hbar} .
$$

Here the WDW patch is defined as the domain of dependence of any Cauchy surface in the bulk whose intersection with the asymptotic boundary is the time slice $\Sigma$.

One of the original evidences supporting the proposal is the linear growth of complexity with respect to time that continues to grow even long after the system reaches thermal equilibrium $[2,3]$. On the other hand for a neutral black hole the growth is bounded by twice of its mass which is sometimes interpreted as the Lloyd's bound on complexity [11]. Moreover if the dual CFT is perturbed, the corresponding change of complexity matches with holographic complexity in the presence of shockwaves [3].

To understand complexity and its holographic dual description, it is important to explore different features of it from both holographic and field theoretic points of view. In

\footnotetext{
${ }^{1}$ Complexity may also be defined for a subregion [6-10].
} 
particular, it is interesting to investigate whether complexity obeys any constraint or any bound such as the Lloyd's bound or not.

Actually despite early observations, it was shown that holographic complexity for the Schwarzschild black hole, which is dual to a thermofield double state, violates the bound [12] (see also [13-15]). More precisely although in the late time regime it approaches a constant value that is twice of the mass of the black hole, the constant is approached from above. More recently the complexity growth for a system underlaying a global quench was also studied in [16] where it was shown that the bound is respected during the growth.

The aim of this paper is to further explore Lloyd's bound in a wider family of states supporting both anisotropic and also hyperscaling violating exponents. Such models admit a fixed point where the physics is invariant under an anisotropic scaling

$$
r \rightarrow \omega r, \quad t \rightarrow \omega^{z} t, \quad x \rightarrow \omega x, \quad d s \rightarrow \omega^{\frac{\theta}{d}} d s
$$

where $z$ and $\theta$ are anisotropic (Lifshitz) and hyperscaling violating exponents. Note that with a non-zero $\theta$, the distance is not invariant under the scaling which in the context of AdS/CFT indicates violations of hyperscaling in the dual field theory. That means in such theories the thermal entropy scales as $S_{\mathrm{th}} \sim T^{\frac{d-\theta}{z}}[17,18]$. Holographically the gravity description of these models may be provided by an Einstein-Maxwell-Dilaton theory (see for example $[19,20])$.

In this paper we will compute the time dependence of holographic complexity for both one and two sided black branes in these models using "complexity $=$ action" proposal. ${ }^{2} \mathrm{We}$ note that the late time behavior of complexity of such models for two sided black branes has been also studied in [21]. For a related study in Lifshitz geometry see [22].

The rest of the paper is organized as follows. In section 2 we present the results corresponding to two sided black branes where we compute the full time behavior of holographic complexity. In section 3 we will study one sided black branes. The last section is devoted to concluding remarks and some related discussions.

\section{Holographic complexity for black branes}

In this section we will compute the on shell action in the WDW patch for black branes with Lifshitz and hyperscaling violating exponents. The model that admits such a solution may be given by a gravitational theory coupled to a gauge field and a scalar field. The corresponding action is [19]

$$
I=\frac{1}{16 \pi G_{N}} \int d^{d+2} x \sqrt{-g}\left(R-\frac{1}{2}(\partial \phi)^{2}+V_{0} e^{\xi \phi}-\frac{1}{4} e^{\eta \phi} F^{2}\right) .
$$

Of course the complete action should have certain Gibbons-Hawking terms defined at spacelike and time-like boundaries. Moreover to accommodate null boundaries it is also crucial

\footnotetext{
${ }^{2}$ There is also another proposal known as "complexity=volume" that we will not consider in this paper. Actually although in this proposal we have linear complexity growth at late times there is no a universal bound for "CV" complexity. Since our main interest is to explore the Lloyd's bound in this paper we will only consider "CA".
} 
to add the corresponding Gibbons-Hawking terms as well as certain joint actions at points of intersection of these null boundaries with any other boundary [23, 24]. Taking all terms into account the action one should consider in the WDW patch is ${ }^{3}$

$$
\begin{aligned}
I= & \frac{1}{16 \pi G_{N}} \int d^{d+2} x \sqrt{-g}\left(R-\frac{1}{2}(\partial \phi)^{2}+V_{0} e^{\xi \phi}-\frac{1}{4} e^{\eta \phi} F^{2}\right)+\frac{1}{8 \pi G_{N}} \int_{\Sigma_{t}^{d+1}} K_{t} d \Sigma_{t} \\
& \pm \frac{1}{8 \pi G_{N}} \int_{\Sigma_{s}^{d+1}} K_{s} d \Sigma_{s} \pm \frac{1}{8 \pi G_{N}} \int_{\Sigma_{n}^{d+1}} K_{n} d S d \lambda \pm \frac{1}{8 \pi G_{N}} \int_{J^{d}} a d S .
\end{aligned}
$$

Here the time-like, space-like, and null boundaries and also joint points are denoted by $\Sigma_{t}^{d+1}, \Sigma_{s}^{d+1}, \Sigma_{n}^{d+1}$ and $J^{d}$, respectively. The extrinsic curvature of the corresponding boundaries are given by $K_{t}, K_{s}$ and $K_{n}$. The function $a$ at the intersection of the boundaries is given by the logarithm of the inner product of the corresponding normal vectors. $\lambda$ parameterizes the null generator of the null boundary which in this paper we use Affine parameterization for the null direction. The sign of different terms depends on the relative position of the boundaries and the bulk region of interest (see [24] for more details).

In what follows we would like to compute the on shell action for black branes with Lifshitz and hyperscaling violating exponents given by

$$
d s^{2}=\frac{L^{2}}{r_{f}^{2 \frac{\theta}{d}}} \frac{1}{r^{2 \frac{d-\theta}{d}}}\left(-\frac{f(r)}{r^{2(z-1)}} d t^{2}+\frac{d r^{2}}{f(r)}+d \vec{x}^{2}\right), \quad A_{t}=\frac{L}{r_{f}^{\frac{\theta}{d}}} \sqrt{\frac{2(z-1)}{d+z-\theta}} \frac{1}{r^{d+z-\theta}}, \quad e^{-\phi}=r^{q}
$$

where $L$ is the radius of the geometry, $r_{f}$ is a dynamical scale where the metric may not be a good description for a UV complete theory above it [26], $q=\sqrt{2(d-\theta)\left(z-1-\frac{\theta}{d}\right)}$ and the parameters of the model are given by

$$
\eta=\frac{2 \theta(d-1)-2 d^{2}}{q d}, \quad \xi=\frac{2 \theta}{q d}, \quad V_{0}=(d+z-\theta-1)(d+z-\theta) \frac{r_{f}^{2 \frac{\theta}{d}}}{L^{2}} .
$$

The function $f(r)$ is also given by

$$
f(r)=1-\left(\frac{r}{r_{h}}\right)^{d+z-\theta}
$$

It is worth mentioning that from null energy condition one has $[19,26]$

$$
(z-1)(d+z-\theta) \geq 0, \quad(d-\theta)(d(z-1)-\theta) \geq 0
$$

Although from these expressions one could have the possibility of $\theta>d$, it was shown that for this case the solution is unstable [26]. Therefore in what follows we consider $d>\theta$ which in turn results to $z \geq 1$.

\footnotetext{
${ }^{3}$ Note that the gauge field is needed to generate an anisotropy for the metric and therefore there is no charge associated to the gauge field. Moreover there is not boundary term for the gauge field. See for example [25].
} 


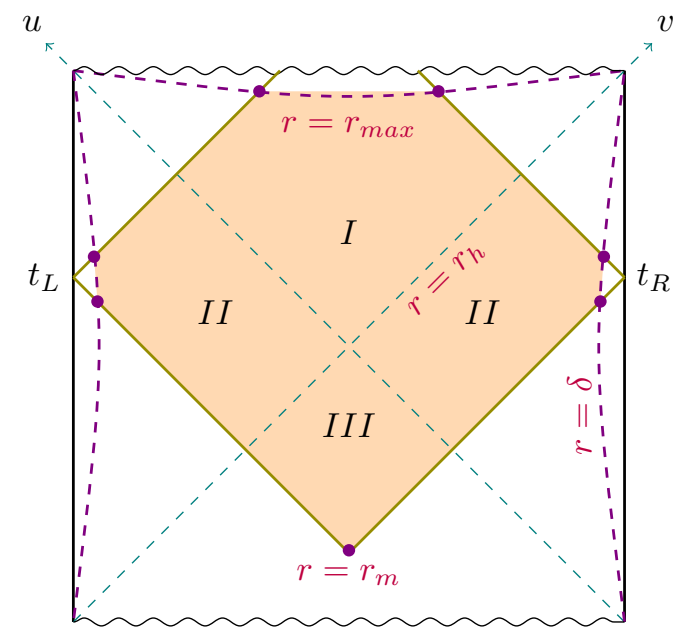

Figure 1. WDW patch of a two sided black brane, moving forward in time assuming $t_{R}=t_{L}$.

It is useful to define an effective dimension $d_{e}=d-\theta$, an effective hyperscaling violating exponent $\theta_{e}=\frac{\theta}{d}$ and also an effective scale $L_{e}=\frac{L}{r_{f}^{\theta / d}}$. Of course in what follows we set $L_{e}=1$. In this notation using the trace of Einstein equation

$$
R-\frac{1}{2}(\partial \phi)^{2}=-\frac{d+2}{d} V(\phi)+\frac{d-2}{4 d} e^{\eta \phi} F^{2},
$$

it is straightforward to see that the action density for the above solution is

$$
\sqrt{-g}\left(R-\frac{1}{2}(\partial \phi)^{2}+V_{0} e^{\xi \phi}-\frac{1}{4} e^{\eta \phi} F^{2}\right)=-2\left(1-\theta_{e}\right)\left(d_{e}+z\right) \frac{1}{r^{d_{e}+z+1}} .
$$

The null boundaries of the right hand side of the WDW patches (see figure 1) we are interested in are given by

$$
t=t_{R}+r^{*}(0)-r^{*}(r), \quad t=t_{R}-r^{*}(0)+r^{*}(r) .
$$

Actually we should admit that computations of the holographic complexity we will be presenting below are very similar to that of Schwarzschild black hole [12]. In particular due to the symmetry of the model the growth rate of complexity is a function of $t_{L}+t_{R}$ and thus for simplicity in what follows we will set $t_{L}=t_{R}=\frac{t}{2}$. Moreover as it was shown [5] (see also [12] ) there is a critical time $t_{c}>0$ below which the growth rate of complexity is zero. Therefore in what follows we well just present the results for $t>t_{c}$.

To proceed, using the notation depicted in figure 1 , for a state at $t_{R}=t_{L}=\frac{t}{2}>\frac{t_{c}}{2}$ one has ${ }^{4}$

$$
\begin{aligned}
I_{I}^{\mathrm{bulk}} & =-2 \times \frac{V_{d}}{8 \pi G_{N}}\left(1-\theta_{e}\right)\left(d_{e}+z\right) \int_{r_{h}}^{r_{\max }} \frac{d r}{r^{d_{e}+z+1}}\left(\frac{t}{2}+r^{*}(0)-r^{*}(r)\right), \\
I_{I I}^{\mathrm{bulk}} & =-2 \times \frac{V_{d}}{4 \pi G_{N}}\left(1-\theta_{e}\right)\left(d_{e}+z\right) \int_{\delta}^{r_{h}} \frac{d r}{r^{d_{e}+z+1}}\left(r^{*}(0)-r^{*}(r)\right), \\
I_{I I I}^{\mathrm{bulk}} & =-2 \times \frac{V_{d}}{8 \pi G_{N}}\left(1-\theta_{e}\right)\left(d_{e}+z\right) \int_{r_{h}}^{r_{m}} \frac{d r}{r^{d_{e}+z+1}}\left(-\frac{t}{2}+r^{*}(0)-r^{*}(r)\right),
\end{aligned}
$$

\footnotetext{
${ }^{4}$ The factor of "2" is a symmetric factor due to the symmetry of the WDW patch.
} 
so that

$$
\begin{aligned}
I^{\text {bulk }}= & -\frac{V_{d}}{2 \pi G_{N}}\left(1-\theta_{e}\right)\left(d_{e}+z\right) \int_{\delta}^{r_{\max }} \frac{d r}{r^{d_{e}+z+1}}\left(r^{*}(0)-r^{*}(r)\right) \\
& -\frac{V_{d}}{4 \pi G_{N}}\left(1-\theta_{e}\right)\left(d_{e}+z\right) \int_{r_{m}}^{r_{\max }} \frac{d r}{r^{d_{e}+z+1}}\left(\frac{t}{2}-r^{*}(0)+r^{*}(r)\right),
\end{aligned}
$$

where $V_{d}$ is the volume of $d$ dimensional subspace of the space time parametrized by $x_{i}, i=1, \cdots d$. Also note that $r_{m}$ can be found from $t=2\left(r^{*}(0)-r^{*}\left(r_{m}\right)\right)$. Note that at the critical time one has $r_{m}=r_{\max }$, so that $t_{c}=2\left(r^{*}(0)-r^{*}\left(r_{\max }\right)\right)$. It is worth mentioning that the time-independent divergent term of the bulk action is

$$
I_{\delta}^{\text {bulk }}=-\frac{V_{d}}{2 \pi G_{N}} \frac{\left(1-\theta_{e}\right)\left(d_{e}+z\right)}{z d_{e} \delta^{d_{e}}} .
$$

There are also several joint actions from which the only one at $r=r_{m}$ has non-zero contribution to the time dependence of complexity. Taking into account the normal vectors of the corresponding null boundaries

$$
k_{1}^{a}=\alpha\left(\frac{r^{2\left(z-\theta_{e}\right)}}{f}\left(\partial_{t}\right)^{a}+r^{z-2 \theta_{e}+1}\left(\partial_{r}\right)^{a}\right), \quad k_{2}^{a}=\beta\left(-\frac{r^{2\left(z-\theta_{e}\right)}}{f}\left(\partial_{t}\right)^{a}+r^{z-2 \theta_{e}+1}\left(\partial_{r}\right)^{a}\right),
$$

the joint action

$$
I^{\text {joint }}=\frac{1}{8 \pi G_{N}} \int d^{d} x \sqrt{\gamma} \log \left|\frac{k_{1} \cdot k_{2}}{2}\right|
$$

reads

$$
I^{\text {joint }}=\frac{V_{d}}{8 \pi G_{N}} \frac{1}{r_{m}^{d_{e}}}\left(\left(z-\theta_{e}\right) \log r_{m}^{2}-\log \left|f\left(r_{m}\right)\right|\right)+\frac{V_{d}}{8 \pi G_{N}} \frac{\log \alpha \beta}{r_{m}^{d_{e}}},
$$

where $\alpha$ and $\beta$ are two constants appearing due to the ambiguity of the normalization of normal vectors of null boundaries. On the other hand from joint points at the surface cut off one gets the following time-independent divergent term

$$
I_{\delta}^{\text {joint }}=-\frac{V_{d}}{4 \pi G_{N}} \frac{\log \alpha \beta \delta^{2\left(z-\theta_{e}\right)}}{\delta^{d_{e}}}
$$

There are also several boundaries that could contribute to the time dependence of the action. We note, however, that the one at the time-like cutoff boundary and the null boundaries do not contribute to the complexity growth rate. The only non-zero contribution comes from the Gibbons-Hawking term at the future singularity at $r=r_{\max }$

$$
I^{\text {surf }}=-2 \times\left.\frac{1}{8 \pi G_{N}} \int d^{d} x d t \sqrt{h} K_{s}\right|_{r=r_{\max }} .
$$

By making use of the fact that

$$
\sqrt{h} K_{s}=-\sqrt{g^{r r}} \partial_{r} \sqrt{h}=-\frac{1}{2} \frac{1}{r^{d_{e}+z-1}}\left(\partial_{r} f(r)-\frac{2\left(d_{e}+z-\theta_{e}\right)}{r} f(r)\right),
$$


one finds

$$
\begin{aligned}
I^{\text {surf }} & =\left.\frac{V_{d}}{8 \pi G_{N}} \frac{1}{r^{d_{e}+z-1}}\left(\partial_{r} f(r)-\frac{2\left(d_{e}+z-\theta_{e}\right)}{r} f(r)\right)\left(\frac{t}{2}+r^{*}(0)-r^{*}(r)\right)\right|_{r=r_{\max }} \\
& =\frac{V_{d}}{8 \pi G_{N} r_{h}^{d_{e}+z}}\left(d_{e}+z-2 \theta_{e}\right)\left(\frac{t}{2}+r^{*}(0)-r^{*}\left(r_{\max }\right)\right) .
\end{aligned}
$$

Since we have used the Affine parametrization for null direction the corresponding boundary term is zero, while form the time like boundary we get the following divergent term

$$
I_{\delta}^{\text {surf }}=\frac{V_{d}}{2 \pi G_{N}} \frac{d_{e}+z-\theta_{e}}{z \delta^{d_{e}}} .
$$

It is also important to note that due to time like boundary there are certain counter terms that needed to make on shell action finite (see e.g. [28]). In the present case these terms lead to the following divergent term [29]

$$
\hat{I}^{\mathrm{ct}}=-\frac{V_{d}}{2 \pi G_{N}} \frac{d_{e}+z-1}{z \delta^{d_{e}}} .
$$

Although this term does not directly contribute to the complexity growth, it is crucial to consider it in order to fully understand the structure of counter terms of the model.

Before evaluating the rate of growth of complexity, we should add proper counter terms to the action in order to remove the ambiguity caused by the normalization of null vectors. Actually the corresponding counter term that does the work has the following form [24] $]^{5}$

$$
\frac{1}{8 \pi G_{N}} \int d \lambda d^{d} x \sqrt{\gamma} \Theta \log \frac{\Theta}{d_{e}},
$$

were $\gamma$ is the determinant of the induced metric on the joint point where two null surfaces intersect, and

$$
\Theta=\frac{1}{\sqrt{\gamma}} \frac{\partial \sqrt{\gamma}}{\partial \lambda}
$$

with $\lambda$ is an affine parameter for the null surface and in the present case and for the null vector $k_{1}$ it is given by

$$
\frac{\partial r}{\partial \lambda}=\alpha r^{z-2 \theta_{e}+1}
$$

For the null surface associated with the null vector $k_{1}$ one finds $\Theta=-\alpha d_{e} r^{z-2 \theta_{e}}$ and thus

$$
\begin{aligned}
I_{1}^{\mathrm{ct}} & =-\frac{1}{8 \pi G_{N}} \int d \lambda d^{d} x \sqrt{\gamma} \Theta \log \frac{\Theta}{d_{e}}=\frac{V_{d} d_{e}}{8 \pi G_{N}} \int_{\delta}^{r_{m}} \frac{d r}{r^{d_{e}+1}} \log \alpha r^{z-2 \theta_{e}} \\
& =-\frac{V_{d}}{8 \pi G_{N}}\left(\frac{\log \alpha r_{m}^{z-2 \theta_{e}}}{r_{m}^{d_{e}}}+\frac{z-2 \theta_{e}}{d_{e} r_{m}^{d_{e}}}\right)+\frac{V_{d}}{8 \pi G_{N}}\left(\frac{\log \alpha \delta^{z-2 \theta_{e}}}{\delta^{d_{e}}}+\frac{z-2 \theta_{e}}{d_{e} \delta^{d_{e}}}\right),
\end{aligned}
$$

\footnotetext{
${ }^{5}$ It is important to note that there is an ambiguity for this equation due to a length scale appearing in the logarithm. Of course since we have already sent the scale to one, there is no a dimensionful scale in this expression, though there is still an ambiguity that we fixed it by the factor of $d_{e}$ in the logarithm. Although we have fixed the factor by hand there is a way to argue how to do that [29].
} 
Similarly for the null surface associated with $k_{2}$ one finds

$$
I_{2}^{\mathrm{ct}}=-\frac{V_{d}}{8 \pi G_{N}}\left(\frac{\log \beta r_{m}^{z-2 \theta_{e}}}{r_{m}^{d_{e}}}+\frac{z-2 \theta_{e}}{d_{e} r_{m}^{d_{e}}}\right)+\frac{V_{d}}{8 \pi G_{N}}\left(\frac{\log \beta \delta^{z-2 \theta_{e}}}{\delta^{d_{e}}}+\frac{z-2 \theta_{e}}{d_{e} \delta^{d_{e}}}\right) .
$$

Therefore altogether one gets

$$
\begin{aligned}
I^{\mathrm{ct}}= & -\frac{V_{d}}{8 \pi G_{N}} \frac{\log \alpha \beta}{r_{m}^{d_{e}}}-\frac{V_{d}}{4 \pi G_{N}} \frac{\log r_{m}^{z-2 \theta_{e}}}{r_{m}^{d_{e}}}-\frac{V_{d}}{4 \pi G_{N}} \frac{z-2 \theta_{e}}{d_{e} r_{m}^{d_{e}}} \\
& +\frac{V_{d}}{8 \pi G_{N}}\left(\frac{\left.\log \alpha \beta \delta^{2\left(z-2 \theta_{e}\right.}\right)}{\delta^{d_{e}}}+\frac{2\left(z-2 \theta_{e}\right)}{d_{e} \delta^{d_{e}}}\right) .
\end{aligned}
$$

Note that there are also other counter terms (from null boundaries extended all the way from cut off surface $\delta$ to $r_{\max }$ ) that result to the following divergent term

$$
\tilde{I}^{\mathrm{ct}}=\frac{V_{d}}{8 \pi G_{N}}\left(\frac{\left.\log \alpha \beta \delta^{2\left(z-2 \theta_{e}\right.}\right)}{\delta^{d_{e}}}+\frac{2\left(z-2 \theta_{e}\right)}{d_{e} \delta^{d_{e}}}\right) .
$$

It is then evident that the ambiguous term drops from the on shell action and we would also get new time dependent terms contributing to the on shell action. Of course it is not the only counter term that could make the on shell action finite. Actually one can see that there are more counter terms needed to make complexity UV finite. ${ }^{6}$ Interestingly enough these new counter terms will also lead to new time dependent terms that have contribution to the complexity growth [29]. To explore the situation let us summarize the terms we have found so far

$$
\begin{aligned}
I^{\mathrm{total}}= & I^{\text {bulk }}+I^{\mathrm{joint}}+I^{\mathrm{surf}}+\hat{I}^{\mathrm{ct}}+I^{\mathrm{ct}}+\tilde{I}^{\mathrm{ct}} \\
= & -\frac{V_{d} \theta_{e}}{2 \pi G_{N}}\left(\frac{\log \delta}{\delta^{d_{e}}}+\frac{1}{d_{e} \delta^{d_{e}}}\right)+\frac{V_{d}}{2 \pi G_{N}} \frac{z-1}{d_{e} \delta^{d_{e}}}+\frac{V_{d}}{4 \pi G_{N}}\left(\frac{\log r_{m}^{\theta_{e}}}{r_{m}^{d_{e}}}-\frac{z-2 \theta_{e}}{d_{e} r_{m}^{d_{e}}}\right) \\
& -\frac{V_{d}}{8 \pi G_{N}} \frac{\log \left|f\left(r_{m}\right)\right|}{r_{m}^{d_{e}}}+\frac{V_{d}}{8 \pi G_{N} r_{h}^{d_{e}+z}}\left(d_{e}+z-2 \theta_{e}\right)\left(\frac{t}{2}+r^{*}(0)-r^{*}\left(r_{\max }\right)\right) \\
& -\frac{V_{d}}{4 \pi G_{N}}\left(1-\theta_{e}\right)\left(d_{e}+z\right) \int_{r_{m}}^{r_{\max }} \frac{d r}{r^{d_{e}+z+1}}\left(\frac{t}{2}-r^{*}(0)+r^{*}(r)\right) \\
& + \text { finite time independent term. }
\end{aligned}
$$

Therefore one needs further counter terms to remove the remaining divergences. Indeed one can see that there are certain counter terms that could remove these divergences and have non-trivial contributions as follows [29] ${ }^{7}$

$$
-\frac{V_{d}}{4 \pi G_{N}}\left(\frac{\log r_{m}^{\theta_{e}}}{r_{m}^{d_{e}}}+\frac{\theta_{e}}{d_{e} r_{m}^{d_{e}}}\right)+\frac{V_{d}}{4 \pi G_{N}} \frac{z-1}{d_{e} r_{m}^{d_{e}}} .
$$

\footnotetext{
${ }^{6}$ We would like to thank the referee for his/her comment that encouraged us to fully address the divergent terms of the model.

${ }^{7}$ Possible counter terms could be [29]

$$
\frac{1}{8 \pi G_{N}} \int d \lambda d^{d} \Sigma \sqrt{\gamma} \Theta\left(\frac{1}{2} \xi \phi+\frac{z-1}{d_{e}}\right) .
$$
}


Taking these terms into account one arrives at

$$
\begin{aligned}
I^{\text {total }}= & -\frac{V_{d}}{4 \pi G_{N}} \frac{1-\theta_{e}}{d_{e} r_{m}^{d_{e}}}-\frac{V_{d}}{8 \pi G_{N}} \frac{\log \left|f\left(r_{m}\right)\right|}{r_{m}^{d_{e}}} \\
& +\frac{V_{d}}{8 \pi G_{N} r_{h}^{d_{e}+z}}\left(d_{e}+z-2 \theta_{e}\right)\left(\frac{t}{2}+r^{*}(0)-r^{*}\left(r_{\max }\right)\right) \\
& -\frac{V_{d}}{4 \pi G_{N}}\left(1-\theta_{e}\right)\left(d_{e}+z\right) \int_{r_{m}}^{r_{\max }} \frac{d r}{r^{d_{e}+z+1}}\left(\frac{t}{2}-r^{*}(0)+r^{*}(r)\right) \\
& + \text { finite time independent term } .
\end{aligned}
$$

Having found all terms contributing to the on shell action, it is then straightforward to compute the growth rate of complexity. Indeed by making use of the fact that $\frac{d r_{m}}{d t}=\frac{f\left(r_{m}(t)\right)}{2 r_{m}^{z-1}(t)}$ one finds

$$
\frac{d}{d t} \mathcal{C}=\frac{1}{\pi} \frac{d}{d t} I_{\mathrm{WDW}}=\frac{2 E}{\pi}\left(1+\frac{d_{e}}{2\left(d_{e}+z-1\right)} \tilde{f}\left(r_{m}(t)\right) \log \left|f\left(r_{m}(t)\right)\right|\right) .
$$

Here

$$
\tilde{f}\left(r_{m}(t)\right)=\left(\frac{r_{h}^{d_{e}+z}}{r_{m}^{d_{e}+z}(t)}-1\right), \quad E=\frac{V_{d}}{16 \pi G_{N}} \frac{d_{e}+z-1}{r_{h}^{d_{e}+z}},
$$

where $E$, at which the complexity approaches at late times, is a parameter that is proportional to the mass of the black brane (see e.g. [27])

$$
M=\frac{d_{e}}{d_{e}+z-1} E .
$$

Thus $E$ reduces to $M$ in the isotropic $(z=1)$ case. For $\theta=0$ the result should be compared with that of $[22] .{ }^{8}$ It is interesting to note that despite the fact that the solution depends on $z$ and $\theta$ exponents, qualitatively the rate of complexity growth behaves the same as that of Schwarzschild black brane [12]. In particular it exhibits a logarithmic divergence at times just after the critical time where $r_{m} \sim r_{\max }$

$$
\frac{d}{d t} \mathcal{C} \sim \frac{2 E}{\pi}\left(1-\frac{d_{e}\left(d_{e}+z\right)}{2\left(d_{e}+z-1\right)} \log \frac{r_{\max }}{r_{h}}\right)
$$

that survives the $\theta=0$ limit.

On the other hand this result shows that Lloyd's bound (defined in terms of the mass of black brane) is always violated for non-trivial anisotropic and hyperscaling violating exponents. This is simply because that the value of $E$, at which the rate of complexity growth saturates to, is always greater than (or equal to) the mass of the black brane $M,{ }^{9}$ which naturally appears on the right hand side of Lloyd's inequality. Of course one may wonder that due to non-trivial scaling of the time coordinate, the Lloyd's bound gets

\footnotetext{
${ }^{8}$ We note, however, that the authors [22] have not considered the counter terms that remove the ambiguity and divergences and therefore their rate of complexity growth has unusual behavior for large $z$. See figure 4 of [22].

${ }^{9}$ Note that from equation (2.6) and with the assumption of $d>\theta$ one has $z \geq 1$.
} 

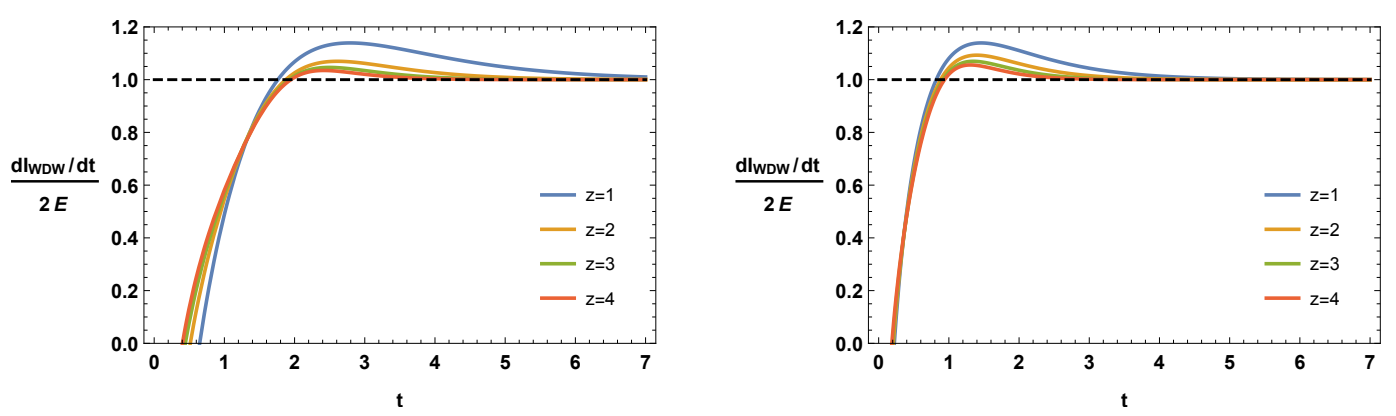

Figure 2. Rate of the complexity growth in a WDW patch for two sided black brane. Left (right) panel shows different values of dynamical exponent for $d_{e}=1\left(d_{e}=2\right)$. For each curve on both panels the Lloyd's bound is violated at some time before the curve reaches unity on the vertical axes.

modified from $2 M$ to a "would be" bound $2 E$. We note, however, that even this "would be" bound is also violated in the present case simply because the rate of complexity growth approaches the bound given by $2 E$ from above (note that $r_{m}(t) \geq r_{h}$ ). More precisely at late times where $r_{m}$ approaches the radius of horizon $r_{h}$ one gets

$$
\frac{d}{d t} \mathcal{C} \sim \frac{2 E}{\pi}\left(1+\frac{d_{e}\left(d_{e}+z\right)}{2\left(d_{e}+z-1\right)}\left(1-\frac{r_{m}(t)}{r_{h}}\right) \log \left(d_{e}+z\right)\left|1-\frac{r_{m}(t)}{r_{h}}\right|\right) .
$$

The behavior of the rate of complexity growth is depicted in figure 2 for different values of $z$ and $\theta$.

It is worth noting that whatever the bound is $(2 M$ or $2 E)$ it is evident from the expression (2.32) that the rate of growth of complexity reaches the bound at a finite time which is of order of $r_{h}$. Of course after this time the complexity still evolves with time till it reaches the bound once again at late times.

By making use of the equation (2.32) one can find the point at which the bound is being saturated. This can be done by setting the time dependent part of the equation (2.32) to zero

$$
\tilde{f}\left(r_{m}\right) \log \left|f\left(r_{m}\right)\right|=0
$$

that solves for $r_{m}=2^{\frac{1}{d_{e}+z}} r_{h}$. Note that it also approaches zero for $r_{m} \rightarrow r_{h}$ that occurs at late times. It is also worth noting that from the explicit form of the blacking function $f(r)$ one gets

$$
t=\frac{2 r_{m}^{z}}{z}{ }_{2} F_{1}\left(1, \frac{z}{d_{e}+z}, 1+\frac{z}{d_{e}+z},\left(\frac{r_{m}}{r_{h}}\right)^{d_{e}+z}\right),
$$

that may be used to read the corresponding time of the special points. In particular for $r_{m} \rightarrow r_{h}$ one has $t \rightarrow \infty$ (late times) and for $r_{m}=0$ one gets $t=0$. Moreover the critical time $t_{c}$ where $r_{m} \rightarrow \infty$ is also given by

$$
t_{c} \sim \frac{1}{4 \sin \frac{z \pi}{d_{e}+z}} \frac{1}{T}
$$

where $T$ is the Hawking temperature of the black hole. 


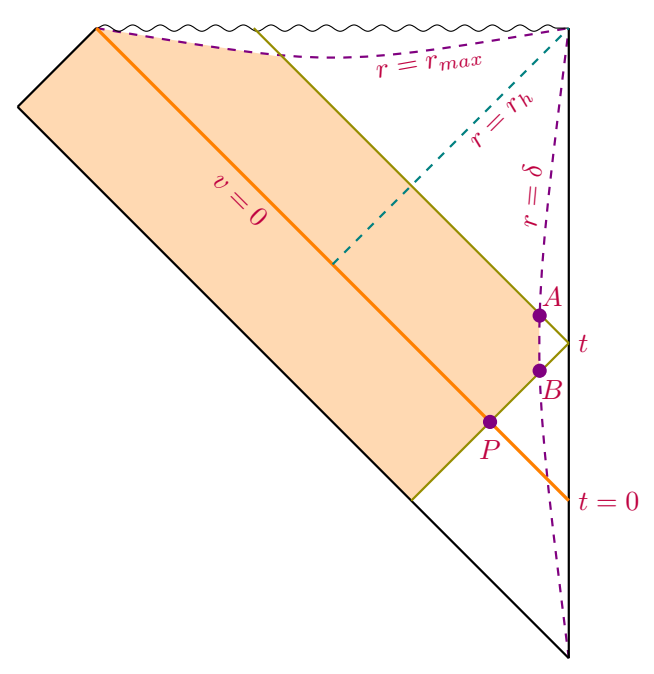

Figure 3. WDW patch of a one sided black brane, i.e., Vaidya geometry. The orange line is the in-falling collapsing null shell located at $v=0$.

\section{Holographic complexity for Vaidya metric}

In this section we will consider holographic complexity for Vaidya geometries with Lifshitz and hyperscaling violating exponents. The model could provide a gravity description for a global quench in a field theory with anisotropic scaling and hyperscaling violation. Adding an infalling null shell matter to the action (2.1), the resultant model admits the following Vaidya metric [30]

$$
d s^{2}=\frac{1}{r^{2\left(1-\theta_{e}\right)}}\left(-\frac{f(r, v)}{r^{2(z-1)}} d v^{2}-\frac{2}{r^{z-1}} d r d v+d \vec{x}^{2}\right), \quad A_{v}=\sqrt{\frac{2(z-1)}{d_{e}+z}} \frac{1}{r^{d_{e}+z}}, \quad e^{-\phi}=r^{\beta},
$$

where

$$
f(r, v)=\left\{\begin{array}{ll}
1 & v<0, \\
1-\left(\frac{r}{r_{h}}\right)^{d_{e}+z} \equiv f(r) & v>0,
\end{array} .\right.
$$

All parameters of the solution are exactly the same as the black brane solution presented in the previous section.

The aim of this section is to compute complexity for the boundary state at time equal to $t$. To do so, one needs to compute the on shell action in the corresponding WDW patch depicted in figure 3. Following [16] we decompose the patch into two parts: $v>0$ and $v<0$ parts (see figure 3).

$\boldsymbol{v}>\mathbf{0}$ part. For $v>0$ we have five boundaries, one at the future singularity and three null boundaries given by $v=0, v=t$ and

$$
t-v=2 \int_{0}^{r_{p}(v, t)} d r \frac{r^{z-1}}{f(r)}
$$


and a time like boundary at $r=\delta$. Using the explicit expression for $f(v, r)$ one finds

$$
t-v=2 \frac{r_{p}^{z}}{z}{ }_{2} F_{1}\left(1, \frac{z}{d_{e}+z}, 1+\frac{z}{d_{e}+z},\left(\frac{r_{p}}{r_{h}}\right)^{d_{e}+z}\right) .
$$

Using the notation of [16] the coordinates of the points $A, B$ and $P$ are determined as follows ${ }^{10}$

$$
\begin{array}{lll}
A: & v_{A}=t, & r_{A}=\delta, \\
B: & v_{B}=t-2 \frac{\delta^{z}}{z}, & r_{B}=\delta, \\
P: & v_{P}=0, & r_{P}=r_{p}(0, t) .
\end{array}
$$

It is then easy to compute the bulk action in this part. Indeed by making use of equation (2.8) one has

$$
\begin{aligned}
I_{v>0}^{\text {bulk }} & =-\frac{1}{8 \pi G_{N}}\left(1-\theta_{e}\right)\left(d_{e}+z\right) \int d^{d} x\left(\int_{0}^{v_{B}} d v \int_{r(v, t)}^{\infty} \frac{d r}{r^{d_{e}+z+1}}+\int_{v_{B}}^{t} d v \int_{\delta}^{\infty} \frac{d r}{r^{d_{e}+z+1}}\right) \\
& =-\frac{V_{d}}{8 \pi G_{N}}\left(1-\theta_{e}\right)\left(\frac{2}{z \delta^{d_{e}}}+\int_{0}^{t-\frac{2}{z} \delta^{z}} \frac{d v}{r^{d_{e}+z}(v, t)}\right) .
\end{aligned}
$$

The $v>0$ part of the WDW has five boundaries three of which are null and their contributions to the on shell action vanish using affine parametrization for the null directions. There is also a time-like boundary at the cutoff surface and one needs to consider the corresponding Gibbons-Hawking term for this boundary. We note, however, that since our ultimate goal is to compute the time dependence of complexity, this term does not contribute to the complexity growth. On the other hand the space-like boundary at the future singularity does indeed contribute to the complexity growth and therefore we will compute the corresponding Gibbons-Hawking term given by

$$
I_{v>0}^{\max }=-\frac{1}{8 \pi G_{N}} \int_{\Sigma_{s}^{d+1}} K_{s} d \Sigma_{s}=-\frac{1}{8 \pi G_{N}} \int d^{d} x \int_{0}^{t} d v \sqrt{\gamma} K_{s} .
$$

From the explicit form of the normal vector to the space-like boundary at the future singularity

$$
n^{a}=-\frac{r_{h}^{\frac{d_{e}+z}{2}}}{r_{\max }^{\frac{d_{e}+z}{2}+\theta_{e}}}\left(\partial_{v}\right)^{a}-\frac{r_{\max }^{\frac{d_{e}+z}{2}}+1-\theta_{e}}{r_{h}^{\frac{d_{e}+z}{2}}}\left(\partial_{r}\right)^{a}
$$

the above Gibbons-Hawking term reads

$$
I_{v>0}^{\max }=\frac{V_{d}}{16 \pi G_{N}} \frac{d_{e}+z-2 \theta_{e}}{r_{h}^{d_{e}+z}} t .
$$

\footnotetext{
${ }^{10}$ Note that in order to fix $v_{B}$ we expand (3.4) at $r_{p} \sim \delta \rightarrow 0$

$$
t-v_{B}=2 \frac{\delta^{z}}{z}{ }_{2} F_{1}\left(1, \frac{z}{d_{e}+z}, 1+\frac{z}{d_{e}+z},\left(\frac{\delta}{r_{h}}\right)^{d_{e}+z}\right) \sim 2 \frac{\delta^{z}}{z} .
$$
}


Finally we need to compute the contributions of the joint points where a null boundary intersects with another boundary (that could also be a null one). The corresponding term is given by the logarithm of the inner product of two intersecting boundaries. To compute such terms one needs to find the normal vectors to the null and time-like boundaries that are given by

$$
\begin{array}{ll}
\text { At } v=0, t & k_{1}^{a}=-\alpha r^{z+1-2 \theta_{e}}\left(\partial_{r}\right)^{a}, \\
\text { At } B P & k_{2}^{a}=\beta\left(\frac{2}{f(r)} r^{2\left(z-\theta_{e}\right)}\left(\partial_{v}\right)^{a}-r^{z+1-2 \theta_{e}}\left(\partial_{r}\right)^{a}\right), \\
\text { At } r=\delta, & k_{3}^{a}=r^{z-\theta_{e}}\left(\partial_{v}\right)^{a}-r^{1-\theta_{e}}\left(\partial_{r}\right)^{a} .
\end{array}
$$

It is straightforward to see that the joint terms at $r=\delta$ (at points $A$ and $B$ ) do not contribute to the time dependence of the on shell action ${ }^{11}$ while the one at point $P$ does. Therefore in what follows we only consider the joint term at pint $P$ where two null boundaries intersect. Indeed by making use of these normal vectors one can find the contribution of the joint point at $P$ as follows

$$
\begin{aligned}
I_{v>0}^{\text {joint }} & =\frac{1}{8 \pi G_{N}} \int_{p} \sqrt{\gamma} d^{d} x \log \left|\frac{k_{1} \cdot k_{2}}{2}\right| \\
& =\frac{V_{d}}{8 \pi G_{N}} \frac{1}{r_{p}^{d_{e}}(t)}\left(\left(z-\theta_{e}\right) \log r_{p}^{2}(t)-\log f\left(r_{p}(t)\right)\right)+\frac{V_{d}}{8 \pi G_{N}} \frac{\log \alpha \beta}{r_{p}^{d_{e}}(t)} .
\end{aligned}
$$

Putting all results together one gets

$$
\begin{aligned}
I_{v>0}= & \frac{V_{d}}{8 \pi G_{N}}\left(1-\theta_{e}\right)\left(\frac{\log \delta}{\delta^{d_{e}}}-\frac{2}{z \delta^{d_{e}}}-\int_{0}^{t-\frac{2}{z} \delta^{z}} \frac{d v}{r^{d_{e}+z}(v, t)}\right)+\frac{V_{d}}{16 \pi G_{N}} \frac{d_{e}+z-2 \theta_{e}}{r_{h}^{d_{e}+z}} t \\
& +\frac{V_{d}}{8 \pi G_{N}} \frac{1}{r_{p}^{d_{e}}(t)}\left(\left(z-\theta_{e}\right) \log r_{p}^{2}(t)-\log f\left(r_{p}(t)\right)\right)+\frac{V_{d}}{8 \pi G_{N}} \frac{\log \alpha \beta}{r_{p}^{d_{e}}(t)} .
\end{aligned}
$$

$\boldsymbol{v}<\mathbf{0}$ part. In this part the function $f(v, t)=1$ and we have four boundaries two of which are given by the past and future horizon and two null boundaries located at $v=0$ and

$$
r^{z}=r_{p}^{z}(t)-\frac{z}{2} v
$$

It is then straightforward to compute the bulk action in this region

$$
\begin{aligned}
I_{v<0}^{\text {bulk }} & =-\frac{1}{8 \pi G_{N}}\left(1-\theta_{e}\right)\left(d_{e}+z\right) \int d^{d} x \int_{-\infty}^{0} d v \int_{\left(r_{p}^{z}(t)-\frac{z}{2} v\right)^{1 / z}}^{\infty} \frac{d r}{r^{d_{e}+z+1}} \\
& =-\frac{V_{d}}{4 \pi G_{N}} \frac{1-\theta_{e}}{d_{e}} \frac{1}{r_{p}^{d_{e}}(t)} .
\end{aligned}
$$

\footnotetext{
${ }^{11}$ The corresponding contribution is
}

$$
S=\frac{V_{d}}{8 \pi G_{N}}\left(\frac{\log \delta}{\delta^{d_{e}}}-\frac{1}{\delta^{d_{e}}}\right) .
$$


The contribution of joint point at $P$ is

$$
I_{v<0}^{\text {joint }}=-\frac{1}{8 \pi G_{N}} \int_{p} \sqrt{\gamma} d^{d} x \log \left|\frac{k_{1} \cdot k_{2}}{2}\right|,
$$

where

$$
k_{1}^{a}=-\alpha r^{z+1-2 \theta_{e}} \partial_{r}, \quad k_{2}^{a}=\beta\left(2 r^{2\left(z-\theta_{e}\right)} \partial_{v}-r^{z+1-2 \theta_{e}} \partial_{r}\right) .
$$

Therefore one finds

$$
I_{v<0}^{\mathrm{joint}}=-\frac{V_{d}}{8 \pi G_{N}} \frac{z-\theta_{e}}{r_{p}^{d_{e}}(t)} \log r_{p}^{2}(t)-\frac{V_{d}}{8 \pi G_{N}} \frac{\log \alpha \beta}{r_{p}^{d_{e}}(t)} .
$$

Since the null boundaries do not contribute to the on shell action, taking both bulk and joint contributions into account one arrives at

$$
I_{v<0}=-\frac{V_{d}}{8 \pi G_{N}} \frac{1}{r_{p}^{d_{e}}(t)}\left(\frac{2\left(1-\theta_{e}\right)}{d_{e}}+\left(z-\theta_{e}\right) \log r_{p}^{2}(t)\right)-\frac{V_{d}}{8 \pi G_{N}} \frac{\log \alpha \beta}{r_{p}^{d_{e}}(t)} .
$$

On shell action and complexity growth. Now we have all ingredients to write the on shell action on the WDW patch for our Vaidya geometry. Indeed one has

$$
\begin{aligned}
I=I_{v>0}+I_{v<0}= & -\frac{V_{d}}{8 \pi G_{N}}\left(1-\theta_{e}\right) \int_{0}^{t-\frac{2}{z} \delta^{z}} \frac{d v}{r^{d_{e}+z}(v, t)}+\frac{V_{d}}{16 \pi G_{N}} \frac{d_{e}+z-2 \theta_{e}}{r_{h}^{d_{e}+z}} t \\
& -\frac{V_{d}}{4 \pi G_{N}} \frac{1}{r_{p}^{d_{e}}(t)}\left(\frac{1-\theta_{e}}{d_{e}}+\frac{1}{2} \log f\left(r_{p}(t)\right)\right) \\
& +(t-\text { independent divergent terms }) .
\end{aligned}
$$

Note that the whole on shell action is independent of $\alpha$ and $\beta$. This is due to the fact that we have used the same free parameters for null vectors for both $v>0$ and $v<0$ regions. Of course one could have considered different parameters, but it can be shown that the result will not change by adding proper counter terms, as that of previous section.

By making use of the differential equation $\frac{d r_{p}(t)}{d t}=\frac{f\left(r_{p}(t)\right)}{2 r_{p}^{z-1}(t)}$ and the fact that the divergent terms are time independent, one can compute the time derivative of the above expression and arrive at

$$
\frac{d}{d t} \mathcal{C}=\frac{1}{\pi} \frac{d}{d t} I_{\mathrm{WDW}}=\frac{2 E}{\pi}\left(1+\frac{1}{2} \frac{d_{e}}{d_{e}+z-1} \tilde{f}\left(r_{p}(t)\right) \log f\left(r_{p}(t)\right)\right),
$$

where

$$
\tilde{f}\left(r_{p}(t)\right)=\frac{r_{h}^{d_{e}+z}}{r_{p}^{d_{e}+z}(t)}-1, \quad E=\frac{V_{d}}{16 \pi G_{N}} \frac{d_{e}+z-1}{r_{h}^{d_{e}+z}},
$$

with $E$ being the value at which the complexity growth saturates at late times. It is worth noting that for $z=1$ the above result reduces to that in [16] with effective dimension $d_{e}$. As we mentioned before the energy of the final black brane, i.e., $M$, is related to $E$ by (2.34). It is then evident that for $z \neq 1$ the Lloyd's bound given by $2 M$ is again violated and the rate of complexity growth saturates at $r_{p}=r_{h}$ to $2 E$. Of course unlike the two 

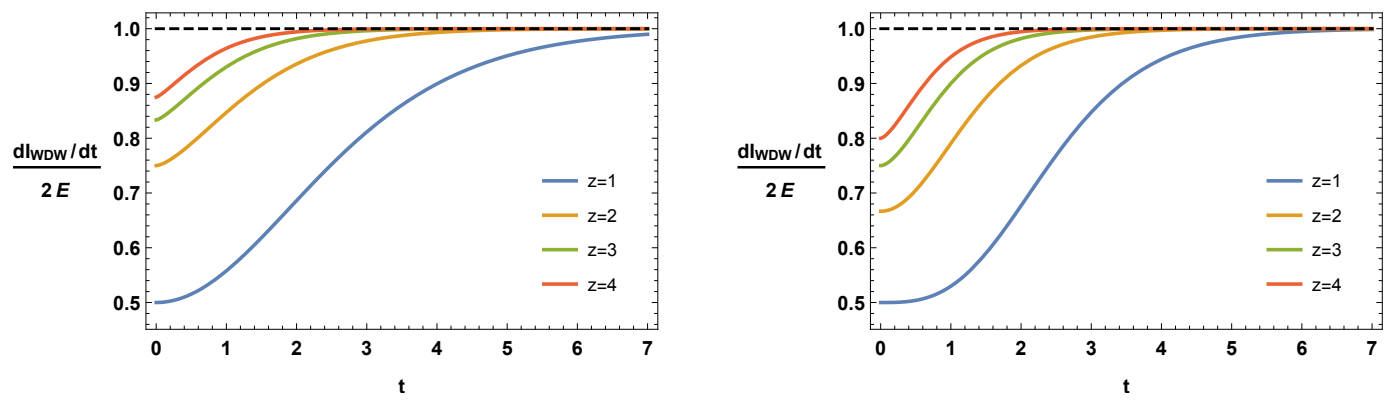

Figure 4. Rate of the complexity growth in a WDW patch for Vaidya geometry with $d_{e}=1$ (left) and $d_{e}=2$ (right).

sided black brane considered in the previous section the "would be" bound $2 E$ is respected in this case. Note that for $z=1$ when the system is isotropic the growth saturates the Lloyd's bound. Also note that for $z \neq 1$ the growth reaches the Lloyd's bound $2 M$ at a finite time which is of order of $r_{h}$.

On the other hand at early time where $r_{p} \sim \delta$ one finds

$$
\frac{d}{d t} \mathcal{C} \sim \frac{2 E}{\pi}\left(1-\frac{1}{2} \frac{d_{e}}{d_{e}+z-1}+\frac{(z / 2)^{1 / z}}{4} \frac{d_{e}}{d_{e}+z-1} \frac{t^{1+\frac{d_{e}}{z}}}{r_{h}^{d_{e}+z}}\right) .
$$

Therefore at early time complexity grows as $t^{1+d_{e} / z}$. The behavior of rate of the complexity growth is depicted in figure 4 which is consistent with the above time dependence growth. In particular for large $z$ complexity grows linearly with time in the early time regime.

\section{Conclusions}

In this paper we have studied complexity growth, using "complexity=action" proposal for a gravitational theory admitting anisotropic black brane solution with non-trivial Lifshitz and hyperscaling violating exponents. We have considered both one and two sided black brane solutions. The two sided black brane would provide a gravitational description for an anisotropic thermofiled double state with hyperscaling violation, while the one sided black brane provides a gravitational description for a global quench in a hyperscaling violating theory.

We have seen that in both cases the Lloyd's bound is violated given the fact that at late times the rate of complexity growth approaches a constant value which is greater than twice the mass of the corresponding black brane. Based on this observation we defined a "would be" bound which is the same as Lloyd's bound but the bound is replaced by the new saturation value. It is however important to note that in the late time regime in one sided black branes this "would be" bound is approached from below, though for that of two sided black branes it is approached from above. This, in turns, means that two sided black brane violates even this "would be" bound. It is also worth noting that for the isotropic case $(z=1)$ the "would be" bound reduces to Lloyd's bound given by twice of the mass. 
As far as the divergent terms are concerned the complexity we have obtained has the following form

$$
\pi \mathcal{C}=I=-\frac{V_{d}}{2 \pi G_{N}}\left(\frac{\log \alpha \beta \delta^{2\left(z-\theta_{e}\right)}}{2 \delta^{d_{e}}}+\frac{1-\theta_{e}}{d_{e} \delta^{d_{e}}}\right)+\text { finite terms },
$$

which for $\theta=0, z=1$ reduces to the complicity associated with an $A d S_{d+2}$ geometry [31]. It is worth noting that from the above volume behavior of the complexity, it looks as the theory lives in an effective dimension $d_{e}$. This is of course consistent with the holographic entanglement entropy of the theory where we have $S \sim \frac{\text { Area }}{\delta^{d_{e}-1}}$ (see for example [32]).

In order to remove the ambiguity associated with the normalization of null vectors one needs to add certain counter terms whose divergent terms are given by

$$
I^{\mathrm{ct}}=\frac{V_{d}}{2 \pi G_{N}}\left(\frac{\log \alpha \beta \delta^{2\left(z-2 \theta_{e}\right)}}{2 \delta^{d_{e}}}+\frac{z-2 \theta_{e}}{d_{e} \delta^{d_{e}}}\right) .
$$

Of course these counter terms are not enough to make the complexity finite. We have seen that there are other counter terms whose divergent terms are

$$
\tilde{I}^{\mathrm{ct}}=\frac{V_{d}}{2 \pi G_{N}}\left(\frac{\log \delta^{2 \theta_{e}}}{2 \delta^{d_{e}}}+\frac{\theta_{e}-z+1}{d_{e} \delta^{d_{e}}}\right) .
$$

It is then clear that all divergences will be dropped resulting to a finite complexity. It is, however, important to note that adding the corresponding counter terms could also have contributions to the finite part of complexity. In particular we have seen that this contribution smooths the early time behavior for large $z$ in two sided black branes. This in turns indicates the importance of the counter terms needed to make the action finite. It would be interesting to explore this point more precisely [29].

We have also seen that the rate of complexity growth reaches the bound at a finite time that is of order of $r_{h}$. We have also evaluated the spatial point $r_{m}$ associated with this time. In particular setting $\theta=0, z=1$, one gets

$$
r_{m}=2^{\frac{1}{d+1}} r_{h} .
$$

Interestingly enough it is the maximal surface that an extremal co-dimension one hypersurface inside the black brane could reach at late times [12].

This means that complexity growth could reach the Lloyd's bound at some time scale comparable to thermalization time of a system, but keep evolving non-trivially after that and finally saturate to the bound in late times. It would be very interesting to explore this behavior more precisely both from gravity and field theory points of view. For recent progress of field theory aspects of complexity see [14, 33-35].

\section{Acknowledgments}

The authors would like to kindly thank M.H. Vahidinia and F. Omidi for useful comments and discussions and also B. Swingle for correspondence. We would also like to thank A. Naseh, A. Shirzad and M. R. Tanhayi for discussions on related topics. We would also like to thank referee for his/her comments. 
Open Access. This article is distributed under the terms of the Creative Commons Attribution License (CC-BY 4.0), which permits any use, distribution and reproduction in any medium, provided the original author(s) and source are credited.

\section{References}

[1] S. Ryu and T. Takayanagi, Holographic derivation of entanglement entropy from AdS/CFT, Phys. Rev. Lett. 96 (2006) 181602 [hep-th/0603001] [INSPIRE].

[2] L. Susskind, Computational complexity and black hole horizons, Fortsch. Phys. 64 (2016) 44 [arXiv: 1403.5695] [INSPIRE].

[3] D. Stanford and L. Susskind, Complexity and shock wave geometries, Phys. Rev. D 90 (2014) 126007 [arXiv:1406.2678] [INSPIRE].

[4] A.R. Brown et al., Holographic complexity equals bulk action?, Phys. Rev. Lett. 116 (2016) 191301 [arXiv: 1509.07876] [INSPIRE].

[5] A.R. Brown et al., Complexity, action and black holes, Phys. Rev. D 93 (2016) 086006 [arXiv: 1512.04993] [INSPIRE].

[6] M. Alishahiha, Holographic complexity, Phys. Rev. D 92 (2015) 126009 [arXiv:1509.06614] [INSPIRE].

[7] O. Ben-Ami and D. Carmi, On volumes of subregions in holography and complexity, JHEP 11 (2016) 129 [arXiv:1609.02514] [INSPIRE].

[8] J. Couch, W. Fischler and P.H. Nguyen, Noether charge, black hole volume and complexity, JHEP 03 (2017) 119 [arXiv: 1610.02038] [INSPIRE].

[9] D. Carmi, R.C. Myers and P. Rath, Comments on holographic complexity, JHEP 03 (2017) 118 [arXiv: 1612.00433] [INSPIRE].

[10] E. Bakhshaei, A. Mollabashi and A. Shirzad, Holographic subregion complexity for singular surfaces, Eur. Phys. J. C 77 (2017) 665 [arXiv: 1703.03469] [InSPIRE].

[11] S. Lloyd, Ultimate physical limits to computation, Nature 406 (2000) 1047 [quant-ph/9908043].

[12] D. Carmi et al., On the time dependence of holographic complexity, JHEP 11 (2017) 188 [arXiv: 1709.10184] [INSPIRE].

[13] M. Ghodrati, Complexity growth in massive gravity theories, the effects of chirality and more, Phys. Rev. D 96 (2017) 106020 [arXiv:1708.07981] [INSPIRE].

[14] R.-Q. Yang, C. Niu, C.-Y. Zhang and K.-Y. Kim, Comparison of holographic and field theoretic complexities for time dependent thermofield double states, JHEP 02 (2018) 082 [arXiv: 1710.00600] [INSPIRE].

[15] M. Moosa, Divergences in the rate of complexification, Phys. Rev. D 97 (2018) 106016 [arXiv: 1712.07137] [INSPIRE].

[16] M. Moosa, Evolution of complexity following a global quench, JHEP 03 (2018) 031 [arXiv: 1711.02668] [INSPIRE].

[17] B. Gouteraux and E. Kiritsis, Generalized holographic quantum criticality at finite density, JHEP 12 (2011) 036 [arXiv:1107.2116] [InSPIRE]. 
[18] L. Huijse, S. Sachdev and B. Swingle, Hidden Fermi surfaces in compressible states of gauge-gravity duality, Phys. Rev. B 85 (2012) 035121 [arXiv:1112.0573] [INSPIRE].

[19] M. Alishahiha, E. O Colgain and H. Yavartanoo, Charged black branes with hyperscaling violating factor, JHEP 11 (2012) 137 [arXiv:1209.3946] [INSPIRE].

[20] A. Salvio, Transitions in dilaton holography with global or local symmetries, JHEP 03 (2013) 136 [arXiv: 1302.4898] [INSPIRE].

[21] B. Swingle and Y. Wang, Holographic complexity of Einstein-Maxwell-dilaton gravity, arXiv: 1712.09826 [INSPIRE].

[22] Y.-S. An and R.-H. Peng, Effect of the dilaton on holographic complexity growth, Phys. Rev. D 97 (2018) 066022 [arXiv: 1801.03638] [INSPIRE].

[23] K. Parattu, S. Chakraborty, B.R. Majhi and T. Padmanabhan, A boundary term for the gravitational action with null boundaries, Gen. Rel. Grav. 48 (2016) 94 [arXiv:1501.01053] [INSPIRE].

[24] L. Lehner, R.C. Myers, E. Poisson and R.D. Sorkin, Gravitational action with null boundaries, Phys. Rev. D 94 (2016) 084046 [arXiv:1609.00207] [INSPIRE].

[25] M. Taylor, Non-relativistic holography, arXiv:0812.0530 [INSPIRE].

[26] X. Dong, S. Harrison, S. Kachru, G. Torroba and H. Wang, Aspects of holography for theories with hyperscaling violation, JHEP 06 (2012) 041 [arXiv:1201.1905] [INSPIRE].

[27] M.H. Dehghani, A. Sheykhi and S.E. Sadati, Thermodynamics of nonlinear charged Lifshitz black branes with hyperscaling violation, Phys. Rev. D 91 (2015) 124073 [arXiv:1505.01134] [INSPIRE].

[28] M. Henningson and K. Skenderis, The holographic Weyl anomaly, JHEP 07 (1998) 023 [hep-th/9806087] [INSPIRE].

[29] A. Akhavan, M. Alsihahiha and F. Omidi, Complexity and counter terms, to appear.

[30] M. Alishahiha, A. Faraji Astaneh and M.R. Mohammadi Mozaffar, Thermalization in backgrounds with hyperscaling violating factor, Phys. Rev. D 90 (2014) 046004 [arXiv: 1401.2807] [INSPIRE].

[31] A. Reynolds and S.F. Ross, Divergences in holographic complexity, Class. Quant. Grav. 34 (2017) 105004 [arXiv: 1612.05439] [INSPIRE].

[32] M. Alishahiha and H. Yavartanoo, On holography with hyperscaling violation, JHEP 11 (2012) 034 [arXiv:1208.6197] [INSPIRE].

[33] R. Jefferson and R.C. Myers, Circuit complexity in quantum field theory, JHEP 10 (2017) 107 [arXiv: 1707 .08570] [INSPIRE].

[34] S. Chapman, M.P. Heller, H. Marrochio and F. Pastawski, Toward a definition of complexity for quantum field theory states, Phys. Rev. Lett. 120 (2018) 121602 [arXiv:1707.08582] [INSPIRE].

[35] R. Khan, C. Krishnan and S. Sharma, Circuit complexity in fermionic field theory, arXiv: 1801.07620 [INSPIRE]. 